\title{
Foot Digit 4
}

National Cancer Institute

\section{Source}

National Cancer Institute. Foot Digit 4. NCI Thesaurus. Code C52842.

The fourth toe from the medial side of the foot. 